\title{
'||||||||||||||||||||||||||||||||||||||||||||||||||||||||||||||||||.
}

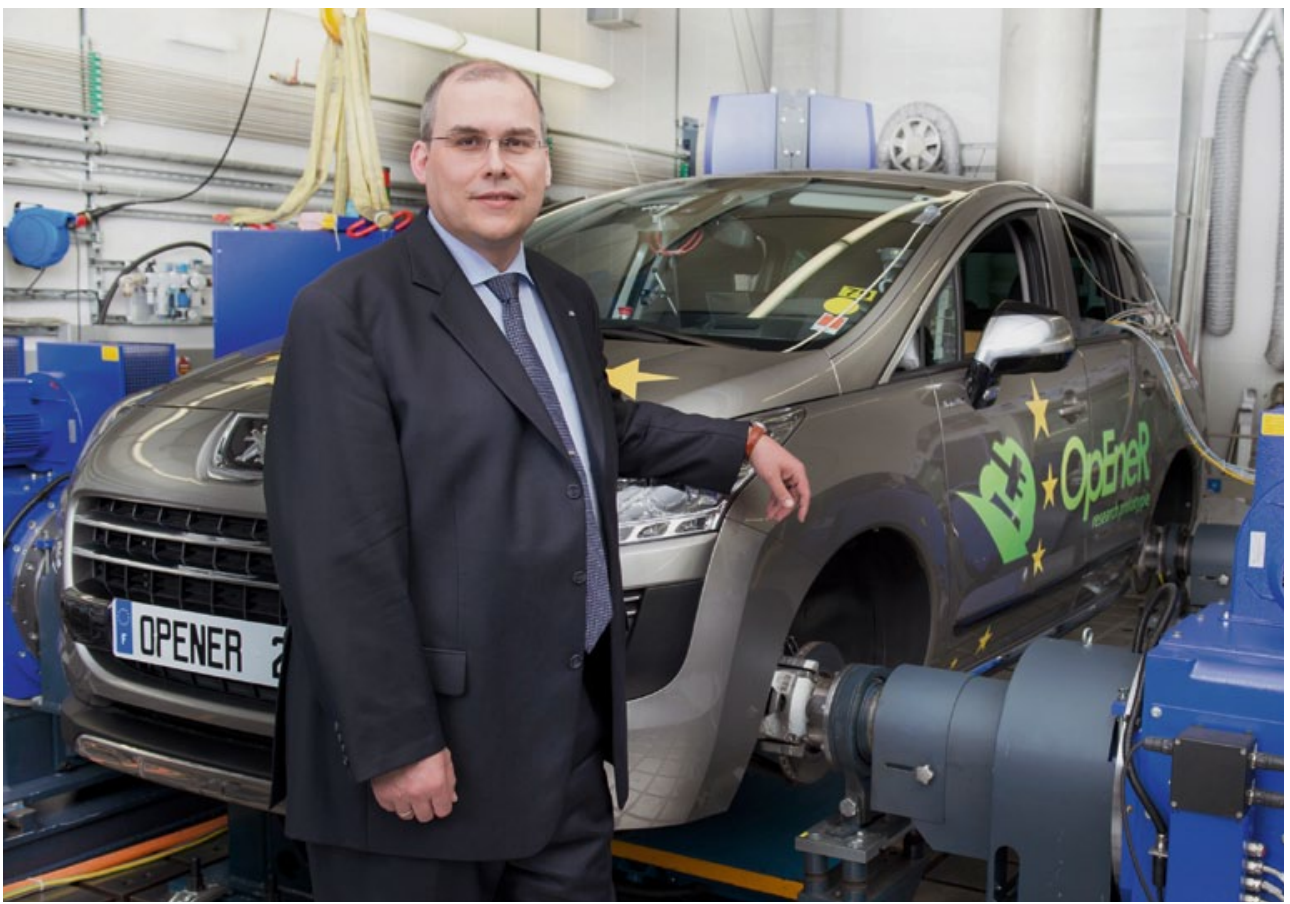

PROF. DR. UWE DIETER GREBE

Geschäftsführer

Global Business Development, Vertrieb \& International Operations AVL List GmbH

\section{DER VERNETZTE ANTRIEB}

Die Fahrzeuge der Zukunft werden deutlich mehr Informationen über den Fahrzustand und die Fahrumgebung zur Verfügung haben. Zahlreiche Sensoren und Systeme vom GPS-basierenden Navigationssystem über Kameras, Ultraschallsensoren und Radar bis hin zu Internetdiensten sind heute bereits in die Fahrzeuge integriert oder werden in der Zukunft verfügbar sein. Die Fahrzeuge werden zunehmend untereinander und mit der Infrastruktur kommunizieren.

Während das Fahrpedal und die Gangwahl in der Vergangenheit die wesentlichen Stellgrößen für das Antriebssystem waren, können nun die zusätzlichen Informationen zur Optimierung der Betriebsstrategie genutzt werden. Dies geschieht bereits für Fahrerassistenzsysteme, die die aktive Sicherheit des Fahrzeugs verbessern. Darüber hinaus eröffnen sich neue Möglichkeiten, Fahrspaß, Verbrauch, Emissionen, Fahrkomfort und Sicherheitsempfinden im realen Fahrbetrieb zu optimieren.

Mit der Kenntnis über Straßenverlauf, Topografie, Geschwindigkeitsbegrenzung, Verkehrssituation und das Verhalten anderer Verkehrsteilnehmer kann das Zusammenspiel der verschiedenen Subsysteme innerhalb des gesamten Antriebsstrangs prädiktiv optimiert werden. Schon bei konventionellen Antrieben lassen sich diese Daten für die ideale Schaltstrategie, das Wärmemanagement oder für intelligente Strategien bei der Abgasnachbehandlung einsetzen. Bei elektrifizierten Antrieben eröffnen sich weitere Möglichkeiten des Energiemanagements. Diese reichen von der Hybridregelstrategie sowie der Bremsaufteilung zwischen Radbremse und der elektrischen Rekuperation bis hin zum Management der Energiespeicherung in der Batterie.

Durch verbesserte Betriebsstrategien können deutliche Reduzierungen im Verbrauch und in den Emissionen im Realfahrbetrieb erzielt werden. Vorausschauende Betriebsstrategien können die Reichweite von Plug-in-Hybriden sowie von batterieelektrischen Fahrzeugen mit oder ohne Range-Extender deutlich steigern. Bei gleicher Reichweite lassen sich die Batterien kleiner dimensionieren, was zu Kostenreduzierungen führt.

Die Steuerung der Energieströme im Fahrzeug muss für den Fahrer möglichst unmerklich geschehen, und das Fahrzeugverhalten muss reproduzierbar bleiben. Vor allem bei Assistenzfunktionen, in denen der Fahrer von der Interaktion mit dem Fahrzeug entkoppelt ist, dürfen die überlagerten Regeleingriffe keinesfalls das Sicherheitsempfinden der Fahrzeuginsassen beeinträchtigen.

Die Beherrschung der zunehmenden Komplexität des vernetzten Antriebsstrangs in Verbindung mit anderen Fahrzeugkomponenten erfordert neue Prozesse und Werkzeuge. AVL erarbeitet neue Funktionalitäten für die Antriebssteuerung und entwickelt Simulationswerkzeuge, Prüfsysteme und Methoden, um alle Potenziale der Konnektivität zusammen mit den Kunden zu erschließen. Durchgängige Entwicklungsumgebungen vom mathematischen Modell bis zur Applikation im Fahrzeug erlauben es, frühzeitig die Zielgrößen Verbrauch, Komfort, Fahrspaß und Sicherheit zu balancieren. 